\title{
Onlineanalyse von Proteinen mittels Ultraschall - der „PROTEINMONITOR“
}

\author{
Stephanie Holz ${ }^{1)}$, Isabelle Schoenbeck ${ }^{1)}$, Marc Stanke ${ }^{5)}$, Michael Hartlep ${ }^{2)}$, Jörg Peters ${ }^{3)}$, \\ Roland Winter ${ }^{4)}$, Sascha Beutel ${ }^{1)}$, Bernd Hitzmann ${ }^{5)}$, Thomas Scheper ${ }^{1)}$ \\ 1) Institut für Technische Chemie, Leibniz Universität Hannover, Hannover, Callinstr. 5, \\ 30167 Hannover \\ 2) TRACE Analytics GmbH, Braunschweig, Richard-Wagner-Str.1, 38106 Braunschweig \\ 3) TF Instruments GmbH, Heidelberg, Im Neuenheimer Feld 583, 69120 Heidelberg \\ 4) Technische Universität Dortmund, Dortmund, Otto-Hahn Str. 6, 44227 Dortmund \\ ${ }^{5)}$ Prozessanalytik und Getreidetechnologie, Universität Hohenheim, Stuttgart, Garbenstr. 25, \\ 70599 Stuttgart
}

\section{Zusammenfassung}

Die Aufreinigung von biotechnologischen Produkten für medizinische Anwendungen stellt zumeist auch auf Grund fehlender Analytik einen Engpass der gesamten Prozesskette dar. Die Optimierung dieser Prozessstufe ist daher von großem Interesse. Ziel ist es durch die Kontrolle der Proteinfaltung Proteine als aktives, hochwertiges Produkt zu erhalten. Ideal dafür ist ein System, das in der Lage ist online die Faltung zu erkennen und an die Downstream-Bedingungen angepasst ist. Ein solches System ist der PROTEINMONITOR, der auf der Ultraschall-Resonator-Technologie (URT) von TF Instruments, Heidelberg, aufbaut. Das bestehende manuelle Messsystem wurde zu einem OnlineMessaufbau umgebaut. Die Messtechnik beruht auf der sehr sensitiven Bestimmung der Ultraschallgeschwindigkeit. Es handelt sich bei dem entwickelten Proteinmonitor um eine nichtinvasive Messtechnik, die mit einem niedrigen Probevolumenstrom von $400 \mu \mathrm{L} / \mathrm{min}$ und ohne Hilfsreagenzien arbeitet. Außerdem ist die Methode flexibel auf jegliches wässrige System anwendbar. Am Beispiel der Aufreinigung von Proteinen aus Kartoffelfruchtwasser mit Hilfe von Membranadsorbern wird die Technologie vorgestellt.

\section{Einleitung}

Die steigende Nachfrage nach biotechnischen Produkten für medizinische Anwendungen, erhöht auch den Bedarf an passender Analytik für den Herstellungsprozess. Die Produktion von Proteinen wird typischerweise in drei Stufen durchgeführt: Upstream, Kultivierung und Downstream. Während dieser Schritte ist es notwendig den Prozess, respektive das Produkt zu verfolgen und zu kontrollieren. Hohe Ausbeuten stehen dabei einer hohen Produktqualität hintenan. Die Aufreinigung der Produkte erfolgt in der Downstream-Stufe. Diese ist sehr kostenintensiv und zeitaufwändig, da es oft an passender Analytik fehlt.

Während der Aufreinigung müssen die Proteine aus der komplexen Kulturbrühe isoliert werden. Dabei werden sie verschiedenen Bedingungen ausgesetzt, wie wechselnden pH-Werten, Temperaturänderungen, verschiedenen Salzgradienten und anderen Cosolventien. Dies kann zu Fehlfaltungen der Proteine führen, was die Proteinaktivität beeinträchtigt. Nur korrekt gefaltete Proteine bilden ein aktives, hochwertiges Produkt. Um die Produktstabilität über die Downstream-Schritte verfolgen zu können, wird ein System benötigt, das in der Lage ist Faltung zu erkennen und unter den gegebenen Bedingungen zu arbeiten.

\section{Das Messsystem}

Die Basis des hier vorgestellten Messsystems PROTEINMONITOR bildet die UltraschallresonatorTechnologie (URT) von TF Instruments, Heidelberg. Das bestehende manuelle Messsystem wurde zu einem Onlinemessaufbau umgebaut. Die Messtechnik beruht auf der sehr sensitiven Bestimmung der Ultraschallgeschwindigkeit mit Hilfe einer Resonatorzelle. 
Die URT nutzt die Resonanz zur präzisen Ultraschallgeschwindigkeitsbestimmung. Durch das Ausbilden einer stehenden Welle über die definierte Strecke $D$ zwischen Generator und Reflektor (Abbildung 1), kann die Ultraschallgeschwindigkeit bestimmt werden. In einem idealen System ist die Resonanzbedingung für jede Welle erfüllt, die bidirektional diesen Raum durchwandert und für die die Gleichung $n \cdot \lambda=2 \cdot D$ erfüllt ist, in der $\mathrm{n}$ eine ganze Zahl darstellt. Die Wellenlänge $\lambda$ bietet Zugang zur Geschwindigkeit $u$ über die Frequenz $f$, da $\lambda \cdot f=u$ ist. Somit kann aus der Frequenz die Geschwindigkeit bestimmt werden.

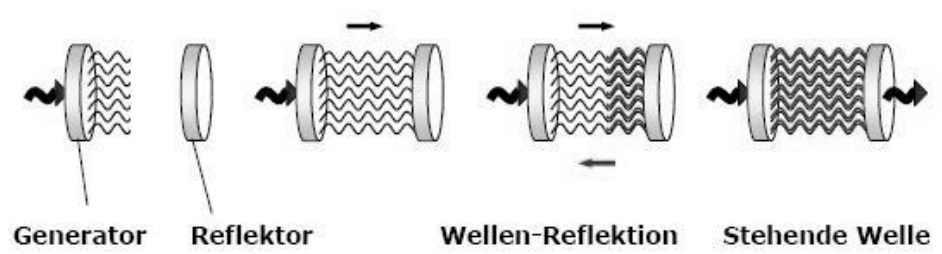

Abbildung 1 Prinzip des Aufbaus einer stehenden Welle in der URT-Messzelle.

Eine stehende Welle bildet sich nur unter den genannten Resonanzbedingungen aus. Weiterhin ist für den Erhalt der Welle ein kontinuierlicher Energieeintrag in das System notwendig, wie es Abbildung 1 zeigt. Durch die Kopplungen zwischen dem Fluid in der Kammer und dem Elektrodenmaterial verhält sich das Messsystem nicht ideal. Aufgrund des anharmonischen Verhaltens ist die direkte Bestimmung der Grundfrequenz $f_{o}$ unmöglich. Daher wird ein spezielles Verfahren nach F. Eggers [3] verwandt, bei dem aus mindestens zehn Resonanzfrequenzen mit einer Ordnung von ca. 70 die Grundfrequenz berechnet wird. Auf diese Weise wird das System für die Messungen kalibriert und bei gleichbleibendem Volumenstrom muss nur eine Resonanzfrequenz für die Geschwindigkeitsbestimmung gemessen werden.

Die URT ist in der Lage Veränderungen einer Proteinprobe zu erkennen und wurde bereits für die Beobachtung von nanopartikularen pharmazeutischen Trägern [2] und Proteinfaltungsvorgängen [8] erfolgreich eingesetzt. Das Messsystem empfiehlt sich dabei durch seine Unabhängigkeit von optischen Eigenschaften der untersuchten Probe, weshalb für das Downstreamprocessing der PROTEINMONITOR entwickelt wurde, der als nichtinvasive Messtechnik, mit einem niedrigen Probevolumenstrom ( $\dot{V}=400 \mu \mathrm{L} / \mathrm{min})$ und ohne Markierung der Proteine, sowie ohne Hilfsreagenzien arbeitet. Außerdem ist er flexibel auf jegliches wässrige System anwendbar. Der Prototyp des PROTEINMONITORS wurde bereits für die Onlineüberwachung von verschiedenen Small Scale Downstream-Schritten getestet. An einem konkreten Beispiel, der Aufreinigung von Proteinbestandteilen aus Kartoffelfruchtwasser, soll im Folgenden die Leistungsfähigkeit des Messsystems, sowie die Vorteile ihrer Anwendung in der Onlineproteinanalyse gezeigt werden.

\section{Kartoffelprotein-Aufreinigung mittels Membranadsorbertechnologie}

Bei der Stärkegewinnung aus Kartoffeln fallen große Mengen an Kartoffelfruchtwasser (KFW) als Nebenstromprodukt an. Dieses enthält bis zu $2 \%$ Protein. Die Proteine können dem KFW durch Membranadsorber entzogen werden. Besonderes Interesse liegt dabei an der Gewinnung der Proteine Patatin und der Gruppe der Protease-Inhibitoren. Patatin ist ein Glykoprotein, das aus 362 Aminosäuren besteht und reich an Lysin und Tyrosin ist. Seine molare Masse beträgt 40-44 kDa. Es zeigt eine Lipid-Acylhydrolase/Transferase-Aktivität [5; 7]. Bei den Protease-Inhibitoren handelt es sich um eine heterogene Klasse hitzestabiler, cysteinreicher Proteine mit einer molaren Masse von 4-23 kDa. Sie dienen den Pflanzen als Schädlingsabwehr [4]. Die natürlichen Eigenschaften dieser Proteine machen sie interessant für Anwendungen in der Medizin oder der Nahrungsmittelbranche [6]. Die spezifische Aufreinigung des Patatins und der Protease-Inhibitoren(PI) erfolgt über Membranadsorber. Der Verlauf der Aufreinigung ist in Abbildung 2 gezeigt. Dabei wird das Bindungsvermögen des Patatins (pI 5,1-9,0) an starke Anionen-Austauscher (Q-Membranadsorber) und der Protease-Inhibitoren (pI 4,5-5,2) an starke Kationen-Austauscher (S-Membranadsorber) ausgenutzt. Das KFW wird in der Anlage direkt über die Adsorber (1) geleitet. Das Waschen der Membranen wird mit $20 \mathrm{mmol} / \mathrm{L}$ Phosphatpuffer durchgeführt (6), der damit ebenfalls keine hohe Salzfracht trägt. Die Rückgewinnung der Proteine (2 und 4) von den Membranen wird mit 0,5 mol/L 
Natriumchlorid-Lösung (3) erreicht. Im anschließenden Regenerationsschritt (5) für die Membranen wird $1 \mathrm{~mol} / \mathrm{L}$ Natriumchlorid-Lösung eingesetzt [1]. Der hohe Einfluss dieser Cosolventien-Fracht auf die Ultraschallgeschwindigkeit muss daher in der Versuchsdurchführung mit dem PPROTEINMONITOR berücksichtigt werden.

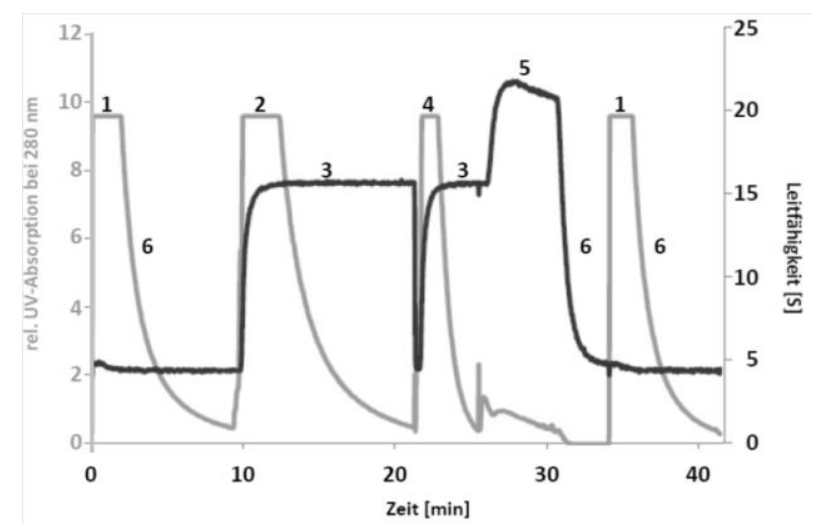

Abbildung 2 Verlauf der einzelnen Schritte während der Aufreinigung der Proteine durch Membranadsorber. Darstellung der gemessenen UV-Absorption (Hellgrau) und der Leitfähigkeit (Dunkelgrau).

Das aufgenommene UV-Signal zeigt mit seinen Maxima an, wann Protein den Prozess verlässt. Die Leitfähigkeit liefert dazu die Information über die Konzentration an Salz, die die Proben enthalten. Protein, das ohne Salzgradienten den Prozess verlässt, wurde nicht über die Membranadsorber gebunden und wird verworfen. Die bisher bei der Aufreinigung eingesetzte Analytik (OnlineBestimmung des Drucks, der UV-Absorption, der Leitfähigkeit und der Trübung) [1] gibt keine Auskunft über die Qualität der gewonnenen Proteinfraktionen. Durch den Einsatz des PPROTEINMONITORS kann somit eine sinnvolle Ergänzung vorgenommen werden. Der Monitor kann dabei auch zum Optimieren der einzelnen Prozessschritte (Beladen, Waschen, Eluieren, Regenerieren) eingesetzt werden.

Aufgrund der Änderungen in der Salzkonzentration wurden zunächst Testläufe an einer FPLC-Anlage (Fast Protein Liquid Chromatrography-Anlage, Bio-Rad, München) vorgenommen. Bei diesem Versuchsaufbau konnte jedoch immer nur ein Membranadsorber genutzt werden. Die folgenden Messungen wurden daher alle mit einem S-Membranadsorber exemplarisch für die Aufreinigung mit Membranadsorbern gemacht. Der Versuchsaufbau wurde dahingehend angepasst, dass nicht der gesamte Prozess, sondern nur die Elutionsproben mittels Ultraschallgeschwindigkeitsanalyse untersucht wurden. Der Versuchsaufbau dafür beruht auf der Rückführung der Elutionslösung in den Vorrat. Über mehrere Prozessläufe reichert sich das Protein in der Elutionslösung immer weiter an.
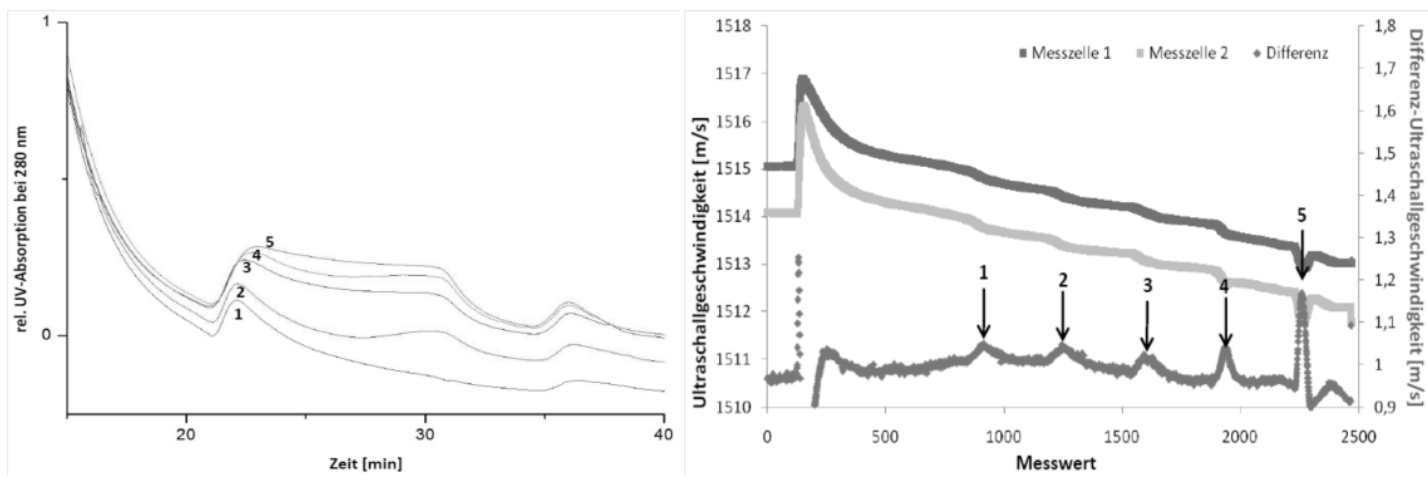

Abbildung 3 Im linken Diagramm sind die gemessenen Daten der UV-Absorption bei der Aufreinigung der Protease-Inhibitoren mittels S-Membranadsorber mit Rückführung des Eluenten zu sehen. Das rechte Diagramm zeigt die dazu erhaltenen Ultraschallgeschwindigkeitsdaten (linke Ordinate) und deren Differenz (rechte Ordinate). 
Die Abbildung 3 zeigt die dazu aufgenommenen Daten mit dem UV-Detektor. Die Zahlen geben die Prozessläufe an. Die Konzentrationserhöhung von 1-5 kann mit dem steigenden UV-Signal, aber auch mit den steigenden Differenzwerten der Ultraschallgeschwindigkeit verfolgt werden.

Auffallend ist das abfallende Grundsignal über die Messung, das nicht in der Differenz wiedergegeben wird, aber beide Messzellen beeinflusst. Es ist durch die Verdünnung des Eluats bei jedem Lauf bedingt. Vor dem Eluieren wird das System mit Puffer gespült. Reste dieses Puffers verbleiben in den Leitungen und vermischen sich beim Eluieren mit dem Eluatstrom. Diese Beobachtung konnte nicht mit der Leitfähigkeitssonde gemacht werden, da diese zu unempfindlich auf Schwankungen in der Salzkonzentration reagiert. Für die Ultraschallmessung ergab sich durch diese Veränderung des Hintergrunds der Probe dank des Ausgleichs über eine Dialyseeinheit keine Beeinträchtigung. Die Messung der Ultraschallgeschwindigkeit als Differenzwert ist so nur möglich, da mit einem Probenund einem Trägerstrom, der durch die Dialyseeinheit dem Probenstrom in allen, außer den Proteinbestandteilen gleicht, gearbeitet wird. In zwei Messzellen werden beim Proteinmonitor diese beiden Ströme parallel vermessen.

\section{Zusammenfassung}

Die Ergebnisse zeigen, dass die Ultraschallmessung zur Bestimmung von Konzentrationsänderungen in der Probe und zum verbesserten Verständnis von Prozessen eingesetzt werden kann. Damit das Primärziel, die Bestimmung der Proteinfaltung für die Proteine aus KFW erreicht wird, bedarf es allerdings noch weiterer Anpassungen des PROZESSMONITORS an diese Aufreinigung.

\section{Danksagung}

Großer Dank gilt dem BMBF, der das Projekt „PROTEINMONITOR“ finanziell gefördert hat und die gesamte Entwicklung, vertreten durch Herrn Zanger, begleitet hat. Ebenso gilt ein großer Dank den Projektpartnern des Projekts und im Besonderen dem Projektkoordinator Herrn Dr. Wolfgang Künnecke.

\section{Literatur}

[1] A.-M. Graf, R. Steinhof, M. Lotz, N. Tippkötter, C. Kasper, S. Beutel and R. Ulber: DownstreamProcessing mit Membranadsorbern zur Isolierung nativer Proteinfraktionen aus Kartoffelfruchtwasser: Chemie Ingenieur Technik 81 (2009) 267-274.

[2] M. Cavegn, R. Douglas, G. Akkermans, and M. Kuentz: Study of an Ultrasound-Based Process Analytical Tool for Homogenization of Nanoparticulate Pharmaceutical Vehicles: Wiley Online Library (2011).

[3] F. Eggers, Model calculations for ultrasonic plate - liquid - plate resonators: peak frequency shift by liquid: Meas. Sci. Technol. 8 (1997) 643-647.

[4] A.J. Hill, S.R. Peikin, C.A. Ryan, and J.E. Blundell: Oral-Administration of Proteinase Inhibitor-Ii from Potatoes Reduces Energy-Intake in Man: Physiology \& Behavior 48 (1990) 241-246.

[5] H.J.H.B. Hirschberg, J.W.F.A. Simons, N. Dekker, and M.R. Egmond: Cloning, expression, purification and characterization of patatin, a novel phospholipase: A. European Journal of Biochemistry 268 (2001) 5037-5044.

[6] L. Pouvreau, H. Gruppen, S.R. Piersma, L.A.M. van den Broek, G.A. van Koningsveld, and A.G.J. Voragen: Relative abundance and inhibitory distribution of protease inhibitors in potato juice from cv. Elkana: Journal of Agricultural and Food Chemistry 49 (2001) 2864-2874.

[7] P.R. Shewry: Tuber storage proteins: Annals of Botany 91 (2003) 755-769.

[8] V. Smirnovas, and R. Winter: Revealing different aggregation pathways of amyloidogenic proteins by ultrasound velocimetry: Biophysical Journal 94 (2008) 3241-3246. 